\title{
SOHO/SUMER observations and analysis of the hydrogen Lyman spectrum in solar prominences
}

\author{
P. Heinzel ${ }^{1,2}$, B. Schmieder ${ }^{2,3}$, J.-C. Vial ${ }^{4}$, and P. Kotrč ${ }^{1}$ \\ 1 Astronomical Institute, Academy of Sciences of the Czech Republic, 25165 Ondřejov, Czech Republic \\ 2 Observatoire de Paris, Section de Meudon, 92195 Meudon Principal Cedex, France \\ 3 Institute of Theoretical Astrophysics, University of Oslo, Blindern, 0315 Oslo, Norway \\ ${ }^{4}$ Institut d'Astrophysique Spatiale, Université Paris XI/CNRS, Bât. 121, 91405 Orsay Cedex, France
}

Received 12 September 2000 / Accepted 9 January 2001

\begin{abstract}
The complete hydrogen Lyman spectrum in several prominences has been observed with the UV spectrometer SUMER on-board the SOHO, during the Joint Observing Programme 107, together with other space and ground-based observatories. Based on these observations, we are able to demonstrate, for the first time, that there exists a large variety of intensities and shapes of Lyman lines in different prominences and in various parts thereof. Therefore, no "canonical" Lyman spectrum can be considered for modelling purposes. However, we have identified at least two representative properties of the observed spectra: in one case (May 28, 1999 prominence) we detected high integrated intensities and no reversals in lines higher than L $\alpha$. Another prominence (June 2, 1999) exhibited quite similar integrated intensities, but all lines have rather strongly reversed profiles. This behaviour cannot be explained in terms of standard isothermal-isobaric models and we thus consider more general models which are in pressure equilibrium with the magnetic field and which have significant prominencecorona transition region (PCTR) temperature gradients. This type of model, recently suggested by Anzer \& Heinzel (1999), is capable of explaining strong emission profiles without reversal. Based on extended non-LTE computations, we suggest that quite different Lyman spectra mentioned above may correspond to two types of PCTRs, one seen along the magnetic-field lines (unreversed profiles) and the other one seen across the field lines (reversed profiles). Finally, we again confirm the importance of partial-redistribution (PRD) scattering processes for Lyman lines in prominences. However, our analysis of new SUMER data also points to a critical role of the $\mathrm{PCTR}$ in radiative transport in these lines.
\end{abstract}

Key words. Sun: prominences - Sun: UV radiation - line: profiles - radiative transfer

\section{Introduction}

Hydrogen lines are the most prominent lines observed in solar prominences, particularly the Balmer $\mathrm{H} \alpha$ line which serves as a standard for prominence imaging. The resonance lines of the Lyman series have also been observed since the Skylab ATM experiment. The LPSP UV-spectrograph on-board the OSO-8 satellite has provided calibrated line profiles of the first two members, $\mathrm{L} \alpha$ and $\mathrm{L} \beta$ (Vial 1982a) and these have been widely used as constraints for non-LTE models of quiescent prominences (Vial 1982b; Heinzel et al. 1987; Paletou et al. 1993; Fontenla et al. 1996). Also, the UVSP on-board the SMM observed prominences in the L $\alpha$ line (Poland \& TandbergHanssen 1983; Fontenla et al. 1988). While the L $\alpha$ line can be usually well reproduced using 1D non-LTE models with the standard partial frequency redistribution (PRD),

Send offprint requests to: P. Heinzel, e-mail: pheinzel@asu.cas.cz the $\mathrm{L} \beta$ intensity was found to be much lower compared to typical OSO-8 data (Vial 1982a; Heinzel et al. 1987). Several studies were then devoted to this problem, trying to increase the computed $\mathrm{L} \beta$ integrated intensity by introducing a prominence-corona transition region (PCTR), multithread structure and the effects of ambipolar diffusion on the energy balance - see summary in Sect. 3 . However, large discrepancies still exist and resolving them is a primary objective of current SOHO/SUMER observations of Lyman lines in quiescent prominences. Another important objective is to study the structure of the base of the PCTR. As discussed previously by Gouttebroze et al. (1993 - GHV), Schmieder et al. (1998) and Schmieder et al. (1999a - SHVR), the Lyman lines are good indicators of the temperature and pressure structure of the lower PCTR, but detailed non-LTE modelling is necessary to infer the proper information. OSO-8 provided only $\mathrm{L} \alpha$ and $\mathrm{L} \beta$ lines, while SHVR have analyzed the higher members observed by SUMER. The latter authors present the 
observations of the intensities of higher-order Lyman lines L5-L9. The GHV-type models were used to compute profiles of these lines and the comparison with observations allowed the determination of certain temperature gradients in the lower part of the PCTR. However, to obtain a complete picture of the base of the PCTR we need observations of the whole Lyman series including $\mathrm{L} \alpha, \mathrm{L} \beta$ and the Lyman continuum. Simultaneously, the central cool parts of the prominence should be diagnosed by using co-temporal and co-spatial observations in optical and infrared lines.

With this rationale in mind and in the framework of the SOHO mission, we have designed a "Joint Observing Programme" - JOP 107, which allows us to observe the whole Lyman series and Lyman continuum with the SUMER spectrograph. JOP 107 also includes CDS rasters in other UV lines and L $\alpha$ imaging by TRACE. During international campaigns, ground-based observatories (GBO) registered spectra and/or images in optical lines. In the present paper we describe these observations made for different prominences. The data show a large variety of intensities and line shapes and this complicates their quantitative interpretation in terms of nonLTE radiative-transfer models. However, even a preliminary analysis of the data clearly shows how they constrain the structure of the PCTR.

In Sect. 2 we present the observations obtained in 1999 for four prominences. In one case the $\mathrm{H} \alpha$ line spectra obtained at the Ondřejov observatory are included. We present the calibrated line intensities for L $\alpha$ to L11, together with the intensities at $907 \AA$ near the head of the Lyman continuum. Section 3 provides a brief summary of the existing non-LTE prominence models, focusing on their predictions of the synthetic Lyman spectra. In Sect. 4 we then show how our observational results compare with the most sophisticated models, in particular we compare the observed Lyman spectra with the predictions of the GHV grid. Section 5 is devoted to non-LTE transfer calculations based on recent magnetohydrostatic models with PCTR (Anzer \& Heinzel 1999 - AH) and we demonstrate the ability of such models to reproduce a large variety of intensities. Finally, Sect. 6 summarizes our results concerning the Lyman spectra and their use for modelling the lower PCTR and points to most important future prospects, both observational and theoretical.

\section{Observations}

SOHO observations of several prominences were performed within the framework of JOP 107 which includes SUMER and CDS spectrographs on-board the SOHO, TRACE satellite and GBO - see Table 1. In the following subsections we briefly describe individual observations.

\subsection{Instruments}

\subsubsection{SUMER}

The SUMER spectrometer on-board the SOHO is described in Wilhelm et al. (1995). In our observations, the SUMER slit $1^{\prime \prime} \times 120^{\prime \prime}$ (slit 4 ) crossed the prominence along the North-South direction. The series of Lyman lines was obtained with an exposure time of $97 \mathrm{~s}$. The spatial resolution along the slit is about $1^{\prime \prime}$. The spectral dispersion is $0.0445 \AA$ per pixel. To be able to observe all the Lyman series lines up to the Lyman continuum we selected four wavelength windows centered around 924, 955, 1010 and $1194 \AA$, respectively (see Table 2). This means that the spectra in these ranges were taken sequentially. Full spectra of around $45 \AA$ are recorded in each window. Strong lines $(\mathrm{L} \beta, \mathrm{L} \gamma, \mathrm{L} \delta)$ are on the bare part of the detector $\mathrm{B}$ and $\mathrm{L} \alpha$ is on the attenuator section. The $\mathrm{L} \alpha$ profile is affected by the attenuator grid. We also observed other transition-region lines: C III triplets at $977.7 \AA$ and $1175 \AA, \quad$ Si III at $1206.5 \AA$, O VI at $1031.9 \AA, \mathrm{S}$ VI at $933.4,944.5,991.8 \AA$, and several chromospheric lines (O I, N I). For data reduction, we destretched the spectra with software of T. Moran (unpublished), corrected them by using the flatfield observed on the day, and calibrated the wavelengths with the software developed at ITA in Oslo. The intensity calibration was performed with the radiometry procedure of Wilhelm et al. (1997). After the temporary loss of $\mathrm{SOHO}$, the radiometric calibration is now accurate to within 30-40\% (see Schühle et al. 2000). For each prominence during the observing time we registered 20 spectra in all the wavelength channels except for the $\mathrm{L} \alpha$ channel where we took only 10 spectra. We averaged the spectra over time except for the March 23, 1999 prominence because of its activity. The instrumental broadening of Lyman lines was neglected.

\subsubsection{CDS}

CDS (Harrison et al. 1995) observed the prominences within a large $240^{\prime \prime} \times 240^{\prime \prime}$ raster in 6 lines (duration $50 \mathrm{~min}$ ) followed by $120^{\prime \prime} \times 240^{\prime \prime}$ rasters in 14 lines (duration $30 \mathrm{~min}$ ). The prominences are seen in emission in the transition-region lines and as dark regions in coronal lines. In coronal line maps (Fe XIII, Si XII, Mg IX, Mg X and $\mathrm{Ca} \mathrm{X}$ ), we observe dark features in the corona over the limb which correspond to the location of the $\mathrm{H} \alpha$ prominence (cool plasma). This is due to the absorption in the $\mathrm{UV}$-continua of $\mathrm{H}$ and $\mathrm{He}$ within the cool prominence material (Kucera et al. 1998).

\subsubsection{TRACE}

The TRACE program consists of observations at $171 \AA$ (Fe IX/X), C IV and L $\alpha$ between 12:00 and 15:00 UT on March 20, 1999 and March 23, 1999 with a field-of-view of $768^{\prime \prime} \times 768^{\prime \prime}$. In $\mathrm{L} \alpha$ the prominence appears to have fine structures moving rapidly, as was observed for other 
Table 1. SUMER observing times for four prominences and characteristics of the coordinated observations

\begin{tabular}{lrrlll}
\hline Date & $\begin{array}{r}\text { Coordinates } \\
\text { SUMER }\end{array}$ & $\begin{array}{r}\text { UT } \\
\text { SUMER }\end{array}$ & $\begin{array}{l}\text { CDS } \\
\text { files }\end{array}$ & $\begin{array}{l}\text { TRACE } \\
\text { channel }\end{array}$ & GBO $\alpha$ spectra \\
\hline March 20 & $434,-842$ & $14: 00-16: 00$ & s15687 & $1216-171-C ~ I V$ & \\
March 23 & $483,-863$ & $12: 00-14: 08$ & s15698 & $1216-171-C$ IV & \\
May 28 & $451,-880$ & $07: 33-09: 39$ & s16214 & & Ondřejov \\
June 2 & $-430,-860$ & $17: 36-19: 54$ & s16359 & & \\
\hline
\end{tabular}

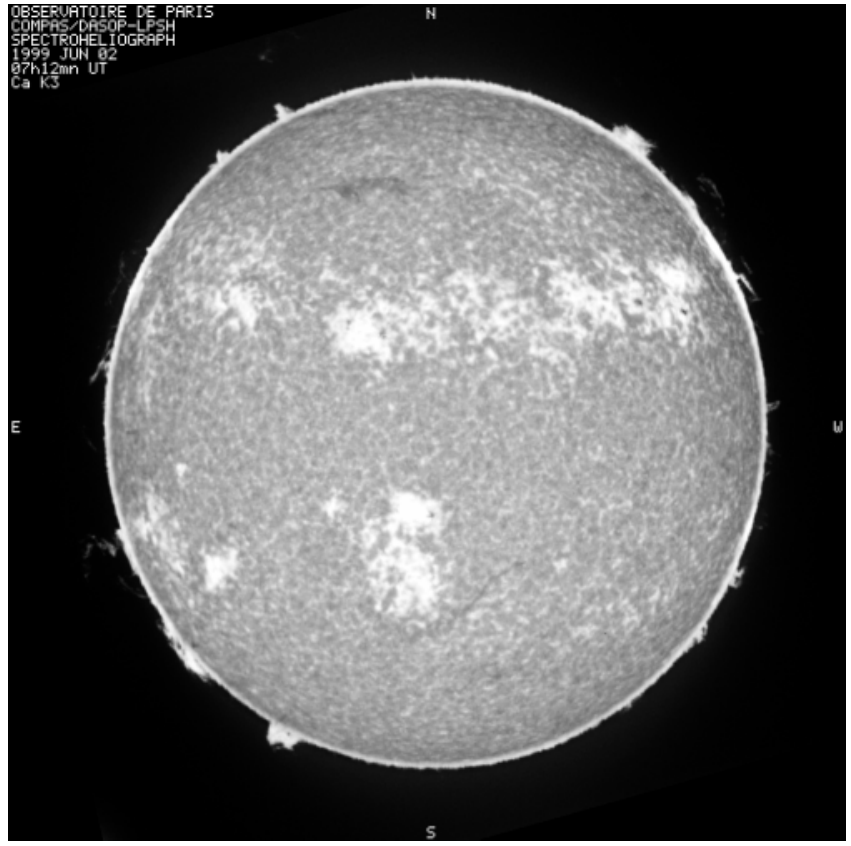

Fig. 1. Prominences of June 2, 1999 (S, E60) and May 28, 1999

prominences with EIT and SUMER (Wiik et al. 1999). At $171 \AA$ we see mainly the absorption of this line by the dense cold material of the prominence at the limb and by the filament in the channel.

\subsubsection{Ground-based observations}

Prominences were observed with the Meudon spectroheliograph in Ca II $\mathrm{K}_{3}$ in the daily routine programme (Fig. 1).

The Ondřejov observatory multichannel spectrograph (Kotrč 1997) took optical spectra and slit-jaw images of the May 28, 1999 prominence, simultaneously with four video cameras, each working with a sampling frequency of 25 pictures per second. The slit of the spectrograph scanned the prominence several times during the observing period, in short sequences lasting about one minute. This allowed us to coalign the slit of the Ondřejov spectrograph with the SUMER slit, within the accuracy limits given by the seeing (which was not better than $2-3^{\prime \prime}$ ). In Fig. 2 we show an example of these spectra (orientation is the same as that of the CDS image shown in Fig. 3). Note that the June 5, 1997 prominence was also observed by the Ondřejov spectrograph (Schmieder et al. 1999c).

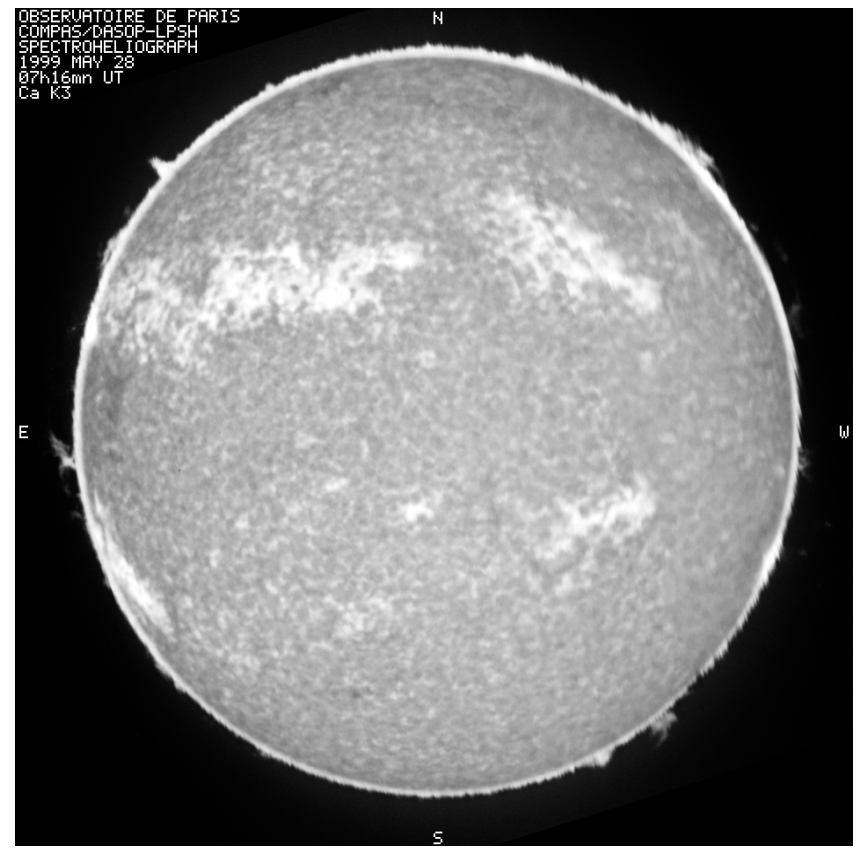

(S, W61) in the Ca II line (Meudon observatory)

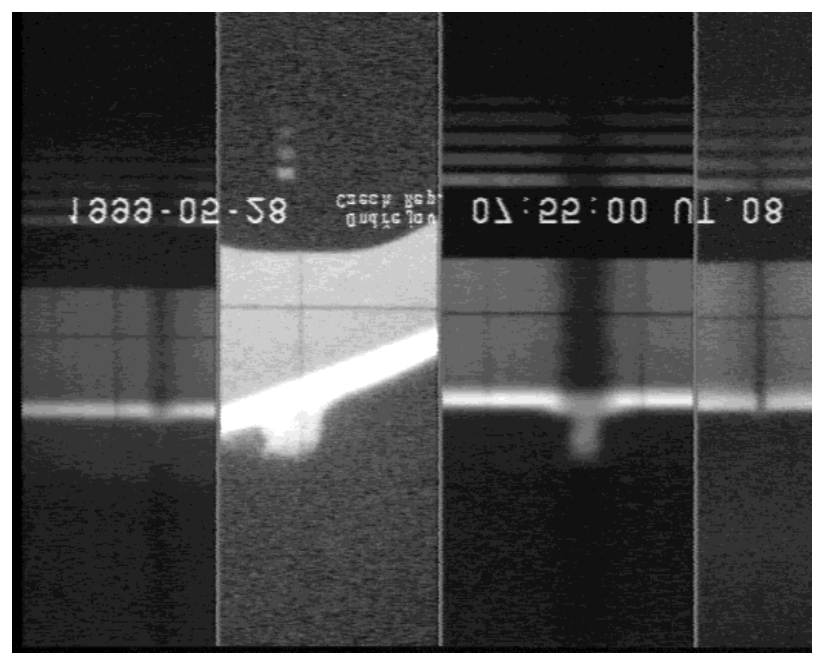

Fig. 2. May 28, 1999 prominence at 07:55:00 UT. Ondřejov CCD video record of the $\mathrm{H} \alpha$ slit-jaw image (second panel) and the spectra in three lines. $\mathrm{H} \alpha$ line is in the third panel, $\mathrm{H} \beta$ (first panel) and Ca II $8542 \AA$ (fourth panel) are weak in this prominence

\subsection{Characteristics of the observed prominences}

We focus our study on the four prominences observed in 1999 (in the following text we will omit the year of 

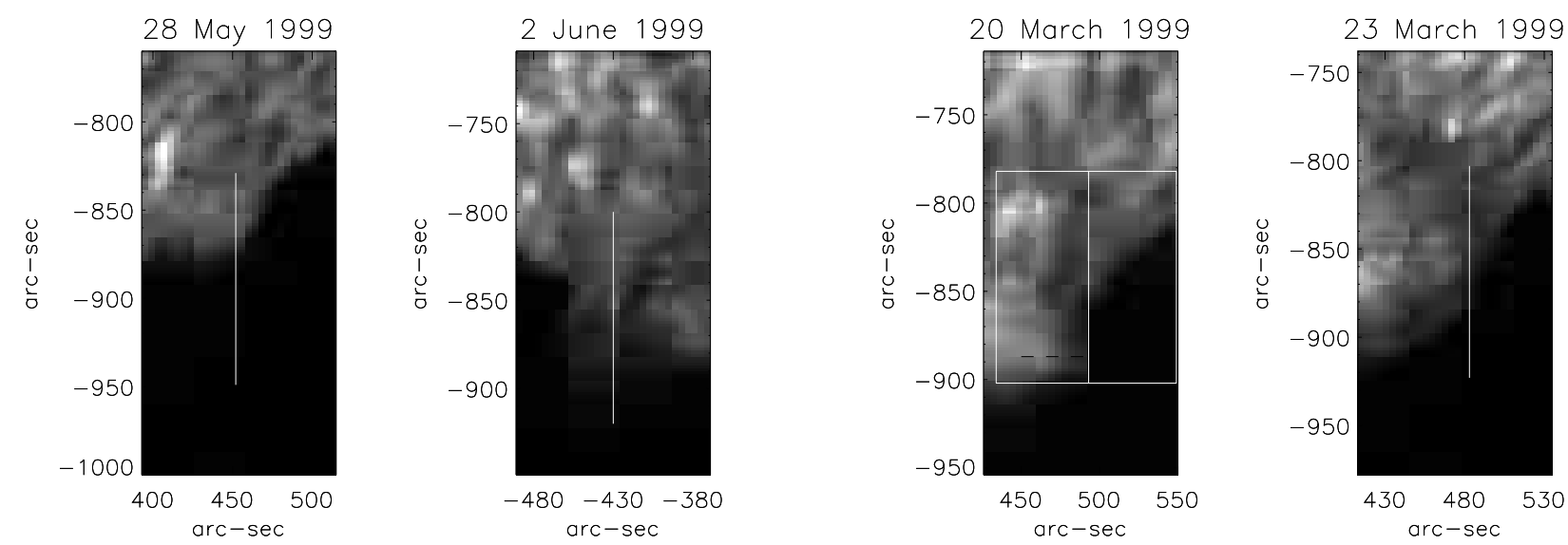

Fig. 3. CDS images in He I of the prominences of May 28, June 2, March 20, March 23 with the SUMER slit position indicated. On the March 20 image, the box represents the raster when the SUMER slit moves from East to West for a context image. The black dashed line shows the location of the pixels of the 6 profiles presented in Fig. 5

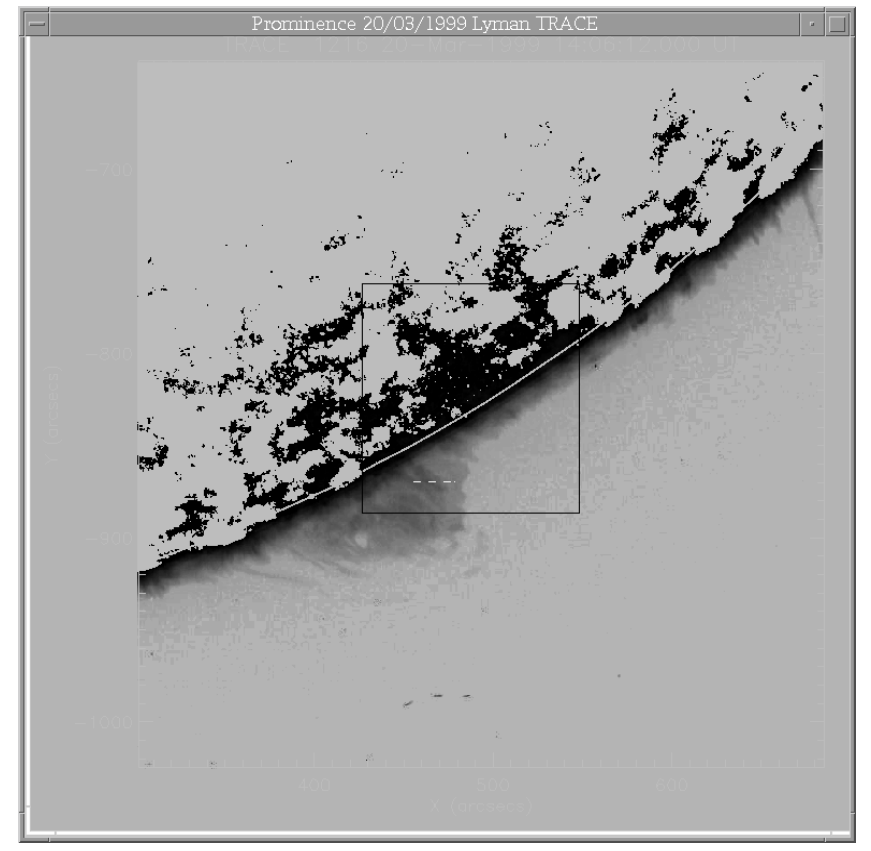

Fig. 4. TRACE image in $\mathrm{L} \alpha$ in inverse gray scale for the prominence of March 20, $1999\left(768 \times 768\right.$ pixels, pixel $\left.=0.5^{\prime \prime}\right)$. The box represents the position of the SUMER raster, the white dashed line the position of the six profiles presented in Fig. 5: the highest intensity corresponds to the Eastern point, the slit crosses two dark structures

observations for brevity) which correspond to polar-crown filaments crossing the limb - see Table 1. On the disk we see the dark filament channel in coronal lines (CDS and TRACE). We have coaligned the SUMER slit position with:

1. CDS He I and optical images (Meudon spectroheliograms or Ondřejov slit-jaw and $\mathrm{H} \alpha$ spectra) for May 28 and June 2 (Figs. 1-3);

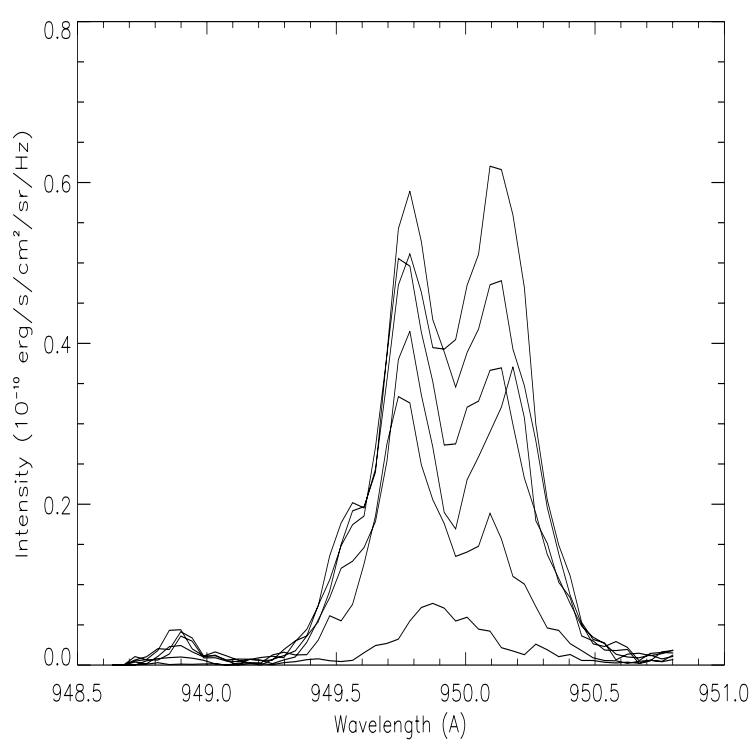

Fig. 5. Variation of L $\delta$ SUMER profiles through the prominence of March 20, 1999. The position of the profiles are indicated by the dashed white line in Fig. 4

\section{TRACE L $\alpha$ image for March 20 and 23 (Fig. 4).}

In two tables we have summarized the results concerning the intensity at the line center and at the peaks for the Lyman series observed in four prominences. In Table 2 we present the central intensity and the peak intensity in prominences, on the disk, on the disk overlaid by filaments and compare them with the values used as the incident radiation in the modelling. Data for the June 5, 1997 prominence are also included for comparison. 
Table 2. Wavelength windows observed by SUMER, covering the Lyman lines and continuum (L $\alpha$ is not shown for prominences because of uncertainties due to the attenuator; but see Fig. 6). A compilation of line-center disk intensities is shown for the reference (Ref), see Heinzel et al. (1997). Intensities of June 5, 1997 prominence (SHVR) are included for comparison. $(P)$ is the specific intensity in the prominence, $(F / D)$ on the disk with a filament, and $(D)$ on the disk. All intensities are in units of $10^{-11} \mathrm{erg} / \mathrm{s} / \mathrm{cm}^{2} / \mathrm{sr} / \mathrm{Hz} . I_{0}$ is the line-center intensity, $I_{\mathrm{p}}$ is the peak intensity

\begin{tabular}{|c|c|c|c|c|c|c|c|c|c|c|}
\hline $\begin{array}{l}\text { Window } \\
\lambda(\AA)\end{array}$ & $\begin{array}{l}\text { Line } \\
\lambda(\AA)\end{array}$ & $\begin{array}{l}\text { Ref } \\
D \\
I_{0} \\
\end{array}$ & $\begin{array}{l}\text { June } 5 \\
P \\
I_{0} I_{\mathrm{p}} \\
\end{array}$ & $\begin{array}{l}\text { March } 23 \\
D \\
I_{0} I_{\mathrm{p}} \\
\end{array}$ & $\begin{array}{l}P \\
I_{0} I_{\mathrm{p}} \\
\end{array}$ & $\begin{array}{l}\text { May } 28 \\
F / D \\
I_{0} I_{\mathrm{p}}\end{array}$ & $\begin{array}{l}P_{\text {dense }} \\
I_{0, \text { range }}\end{array}$ & $\begin{array}{l}\text { June } 2 \\
F / D \\
I_{0} I_{\mathrm{p}} \\
\end{array}$ & $\begin{array}{l}P \\
I_{0, \text { range }}\end{array}$ & $I_{\mathrm{p}, \text { range }}$ \\
\hline $1172-1216$ & $1215.67 \mathrm{~L} \alpha$ & 2900 & & & & & & & & \\
\hline 988-1033 & $1025.72 \mathrm{~L} \beta$ & 42.6 & & 4060 & 1325 & 2550 & $40-70$ & 2555 & $12-17$ & $15-33$ \\
\hline \multirow[t]{3}{*}{ 933-978 } & $972.54 \mathrm{~L} \gamma$ & 9.78 & & 1012 & 3.24 .5 & 710 & $10-14$ & 610 & 4 & $10-13$ \\
\hline & $949.74 \mathrm{~L} \delta$ & 4.32 & 1.82 .8 & 4.25 .5 & 1.92 .5 & 3.54 .5 & $3-6$ & & $1.5-2$ & $3-4$ \\
\hline & $937.80 \mathrm{~L} \epsilon$ & 3.10 & 1.12 & 2.53 .0 & 1.4 & 2.53 .0 & 2.5 & 1.73 .0 & 1.7 & 3 \\
\hline \multirow[t]{7}{*}{$901-946$} & 930.75 L6 & 2.17 & 0.91 .4 & 2.3 & 0.95 & 1.71 .8 & 1.8 & 1.41 .9 & 1.5 & 2 \\
\hline & $926.20 \mathrm{~L} 7$ & 1.86 & 0.71 .0 & 1.8 & 0.7 & 1.41 .5 & 1.2 & 1.11 .4 & 1.2 & 1.4 \\
\hline & 923.15 L8 & 2.02 & & 1.9 & 0.75 & 1.51 .8 & 1.2 & 1.6 & 1.5 & \\
\hline & 920.96 L9 & 1.49 & & 1.3 & 0.55 & 1.1 & 0.9 & 1.1 & 1.1 & \\
\hline & 919.35 L10 & 1.40 & & 1.2 & 0.5 & 1.1 & 0.8 & 0.95 & 0.9 & \\
\hline & 918.13 L11 & 1.40 & & 1.15 & 0.5 & 1.1 & 0.7 & 0.95 & 0.9 & \\
\hline & 907 cont & 1.48 & & 0.9 & 0.45 & & 0.61 & 0.65 & 0.45 & \\
\hline
\end{tabular}

Table 3. Mean integrated intensities for different structures $\left(\mathrm{erg} / \mathrm{s} / \mathrm{cm}^{2} / \mathrm{sr}\right)$. These values differ somewhat from those shown in Figs. 6 and 7 because of different locations and averaging

\begin{tabular}{llllllllll}
\hline \multirow{2}{*}{ Structure } & Pixels & Date & $\mathrm{L} \alpha$ & $\mathrm{L} \beta$ & $\mathrm{L} \gamma$ & $\mathrm{L} \delta$ & $\mathrm{L} \epsilon$ & $\mathrm{L} 6$ & $\mathrm{H} \alpha$ \\
& & & & & & & & & \\
\hline disk & $107-117$ & March 23 & 116000 & 850 & 200 & & & & \\
& $1-30$ & May 28 & 114000 & 900 & 225 & 120 & 65 & & \\
filament & $1-90$ & June 2 & 76000 & 780 & 210 & & & & \\
& $70-117$ & March 23 & 73500 & 600 & 140 & & & & \\
\hline prominence & $41-50$ & May 28 & 103000 & 700 & 175 & 80 & 40 & 25 & $1.3+5$ \\
(close to limb) & $51-61$ & & 102000 & 640 & 120 & 65 & 25 & & $1.0+5$ \\
prominence & $45-65$ & March 23 & 37000 & 310 & 75 & 40 & 20 & 15 & \\
& $40-45$ & & 28300 & 220 & 45 & & & & \\
bubble & $20-40$ & & 11050 & 80 & & & & & \\
prominence & $80-100$ & June 2 & 46200 & 530 & 165 & 90 & 40 & 25 & \\
(faint) & $100-110$ & & 27400 & 220 & 40 & & & & \\
edge & $92-103$ & March 20 & & 120 & 30 & 15 & & & \\
\hline
\end{tabular}

In Table 3 we present the integrated intensity for different parts of the prominences, commonly 10 pixels mean, while the profiles presented in Figs. 5-8 are averaged over five pixels.

\section{March 20}

Rasters in the $\mathrm{L} \delta, \mathrm{L} \epsilon, \mathrm{S}$ VI $(933.378 \AA)$ lines were performed during the observation for context images. The SUMER slit scanned part of the prominence with a step of $3^{\prime \prime}$ (Fig. 4). The prominence extension is $120^{\prime \prime}$ along the limb and the height at the maximum is about $60^{\prime \prime}$. The raster was not perfectly centered on the prominence so we missed a large part of it. Nevertheless, we have a good sample of profiles from the center to the edge (Fig. 5). The altitude of the registered profiles in Fig. 5 is between 10000 and $15000 \mathrm{~km}$. L $\delta$ has no reversal for small intensities, it is reversed for higher intensities, and the reversal is again less pronounced at highest intensities. The SUMER slit for temporal series was located at the edge of the prominence with low intensities.

\section{May 28}

This prominence is low (14000 km) and dense (Figs. 2, 3). There are about 30 pixels on the slit crossing the prominence $\left(30^{\prime \prime}\right)$ and 30 points on the disk which is covered by a filament. The thickness of the prominence is so large that there is little difference between the intensity of the filament and the prominence close to the limb. In Figs. 6-8 we present mean profiles of the Lyman series in this prominence. Apart from $\mathrm{L} \alpha$, the profiles have no central reversal.

\section{June 2}

The prominence is rather extended (Fig. 3). Twenty pixels 

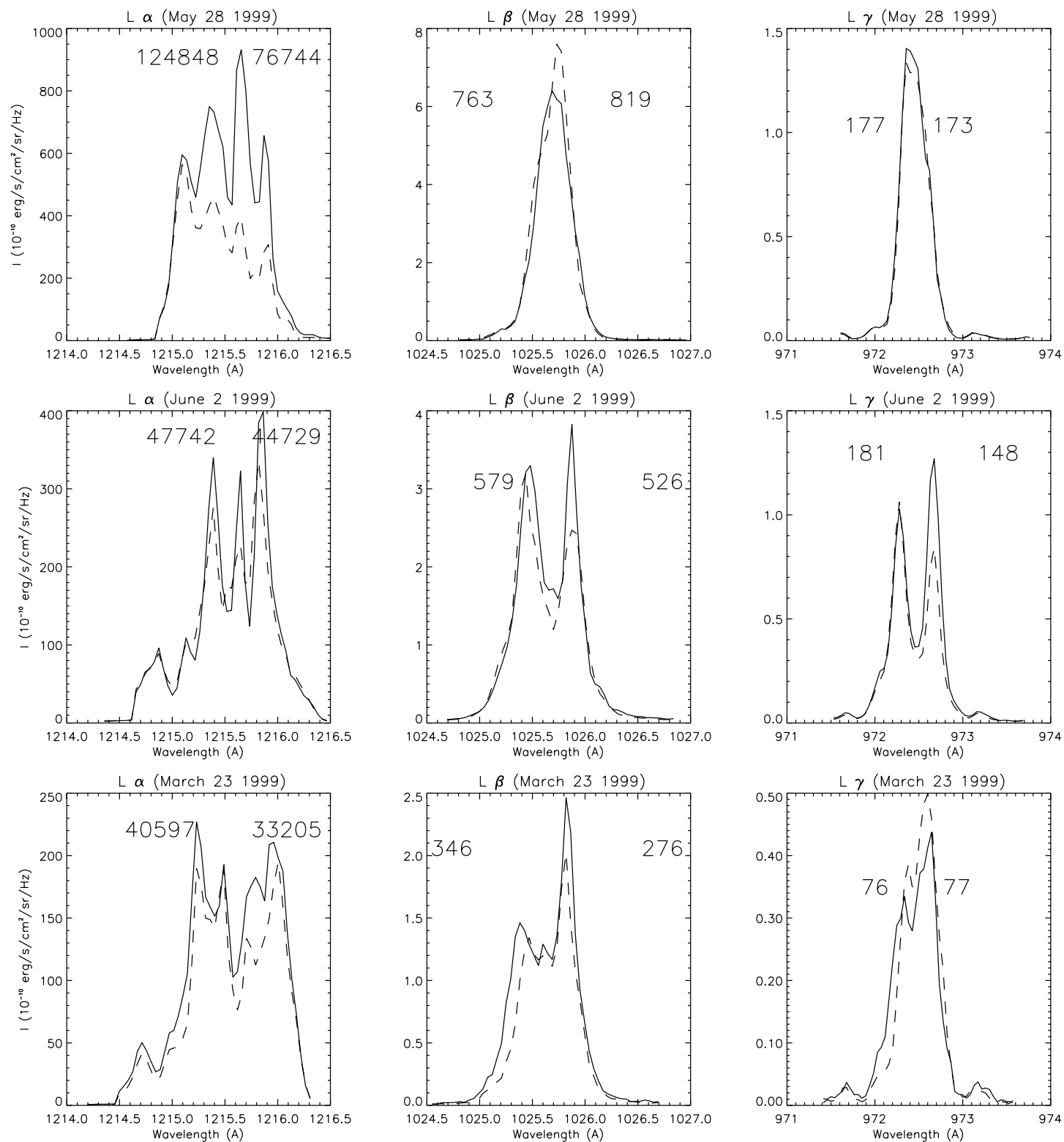

Fig. 6. Profiles of the $\mathrm{L} \alpha, \mathrm{L} \beta$ and $\mathrm{L} \gamma$ lines through three prominences (mean over 5 pixels along the slit, full and dashed profiles correspond to different spatial positions). The numbers in each panel are the integrated intensities of the two profiles in $\mathrm{erg} / \mathrm{s} / \mathrm{cm}^{2} / \mathrm{sr}$ (left/right number corresponds to the full/dashed line profile, respectively)

(i.e. $20^{\prime \prime}$ ) have a large intensity, while ten others have few counts; we refer to this part as a faint prominence in Table 3. Figures 6-8 present mean profiles of Lyman series in the prominence. The integrated intensities are similar to those of the March 28 prominence, but the profiles are strongly reversed.

\section{March 23}

The prominence is quite active and it is not meaningful to integrate the spectra over time. We reduced the first 8 spectra (Schmieder et al. 1999b). Along the slit we observed the disk (70:120 pixels) and the prominence (45 pixels) which consists of three parts:
A - dense part (54-69);
B - highly redshifted part (38-41);
C - faint bubble (24-27).

In Figs. 6-8 we show examples of line profiles obtained in part $\mathrm{A}$ of the prominence. The disk is mainly overlaid by a filament; only a few points (107-117) represent the true disk. Preliminary results for this prominence were presented in Schmieder et al. (1999b). The new calibration (Schühle et al. 2000) leads to intensity values smaller by $10-15 \%$ as compared to the previous ones obtained with the calibration of 1997 . 

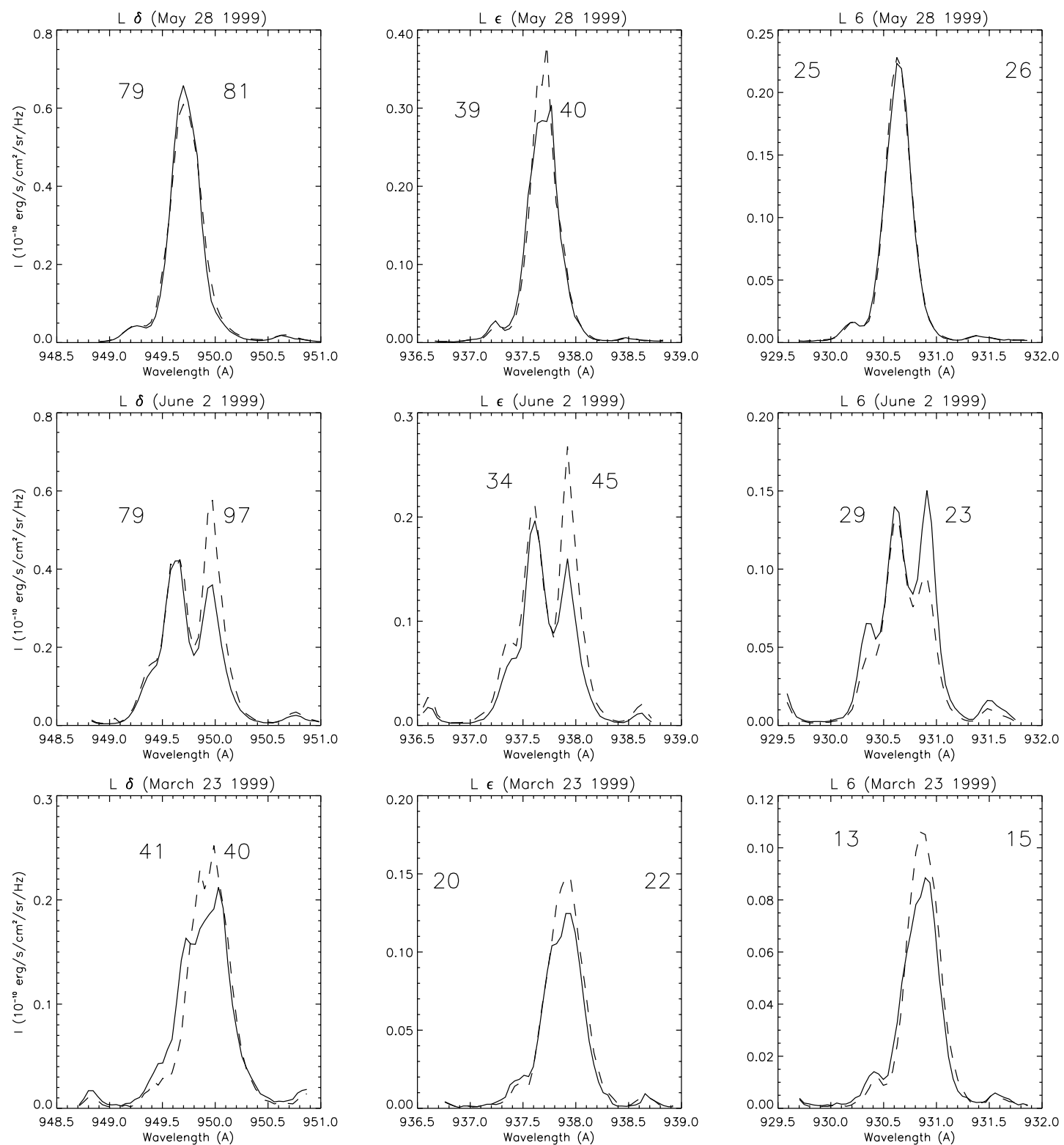

Fig. 7. Profiles of the $\mathrm{L} \delta, \mathrm{L} \epsilon$ and $\mathrm{L} 6$ lines through three prominences (see Fig. 6)

\section{Theoretical non-LTE models predicting the Lyman-spectrum intensities}

The first detailed multilevel non-LTE prominence models were devised by Heasley, Mihalas, Milkey and Poland (see Heasley \& Milkey 1978 and references therein). L $\alpha$ and $\mathrm{L} \beta$ lines were computed assuming the complete frequency redistribution (CRD) taking place during the scattering process. At those times, no observed profiles were available for comparison and these models were mainly aimed at studying the optical lines of hydrogen, helium and Ca II. Later, Heasley \& Milkey (1983), using the same models, studied the Lyman continuum.

Vial (1982b) extended this modelling to 2D, but still using CRD and only a two-level atom model (this was dictated by enormous demands on CPU time for 2D models). However, he was able to demonstrate relatively good agreement between theoretical $\mathrm{L} \alpha$ intensities and those observed by OSO-8, apart from certain discrepancies in the line shapes. Later these 2D models were generalized by Paletou (1995) who used a multilevel model atom and PRD for $\mathrm{L} \alpha$ and $\mathrm{L} \beta$.

The first consistent treatment of partial frequency redistribution in $\mathrm{L} \alpha$ and $\mathrm{L} \beta$ was presented by Heinzel et al. (1987), in the framework of 1D isothermal-isobaric models of Heasley \& Milkey (1978). A new ingredient, which has been shown to be critical for calculating the correct line shapes within PRD, was the introduction of realistic (i.e., observed) incident line intensities. The basic results of this study were: (i) large differences between $\mathrm{L} \alpha$ intensities and profile shapes computed with PRD and with CRD, (ii) for low-temperature models, the central intensity of $\mathrm{L} \alpha$ is practically fixed at the value of the diluted incident chromospheric intensity. This applies for both PRD 

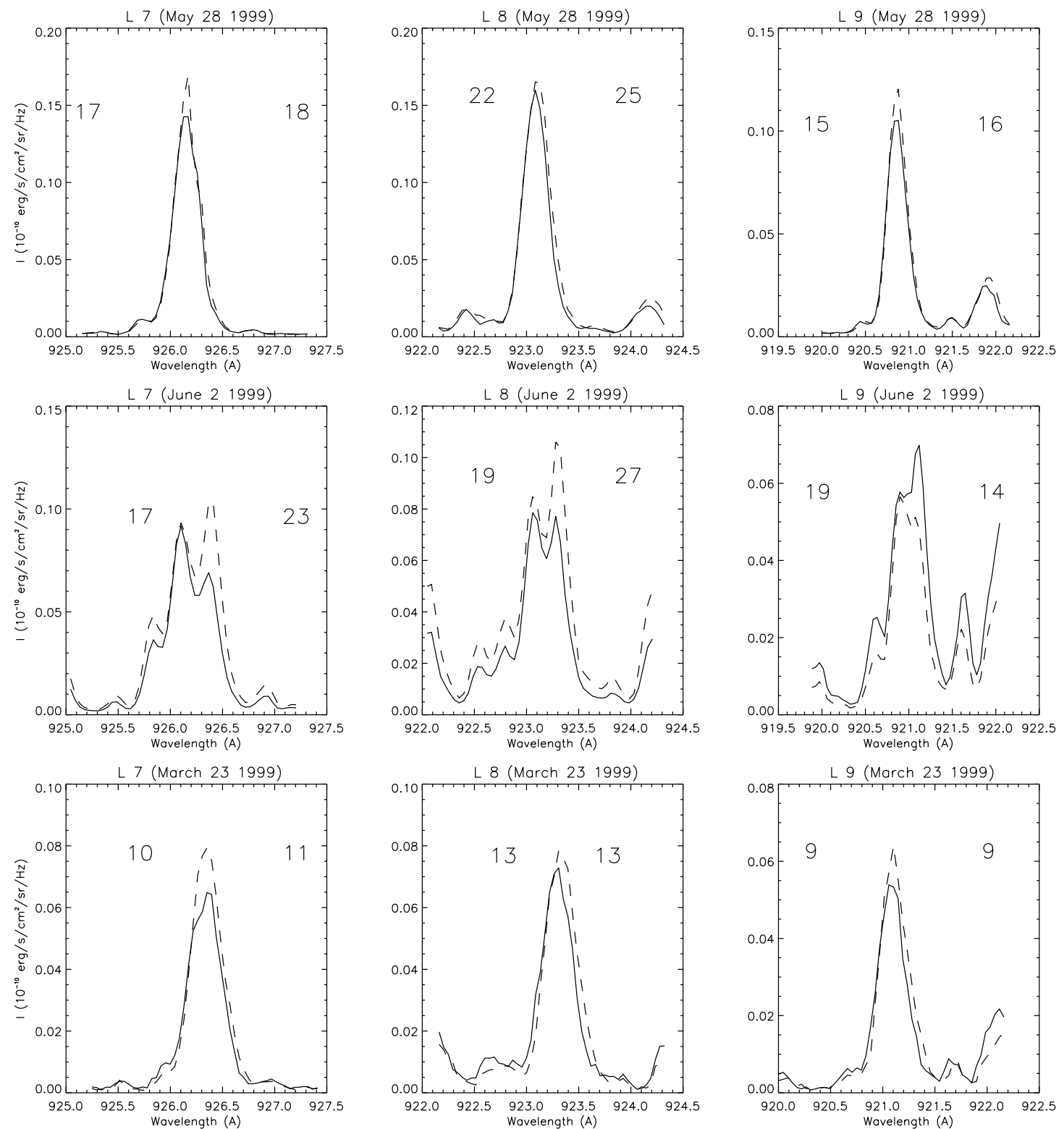

Fig. 8. Profiles of the L7, L8 and L9 lines through three prominences (see Fig. 6)

and CRD (however, the wings differ markedly between PRD and CRD), (iii) a comparison of the computed $\mathrm{L} \alpha$ with OSO-8 observations of one particular prominence led to excellent agreement, while the $\mathrm{L} \beta$ integrated intensity was found to be too low by a factor of more than five.

The problem of the $\mathrm{L} \beta$ integrated intensity was then studied by Heinzel et al. (1988), who introduced a schematic PCTR into the standard 1D models. This led to certain enhancements of $\mathrm{L} \beta$, but the quantitative comparison between computed and observed (OSO-8) $\mathrm{L} \alpha$ and $\mathrm{L} \beta$ was still rather problematic. However, as we will see later, the concept of the PCTR in modelling the Lyman line intensities is very crucial.

At the Hvar IAU Coll. 119, Vial et al. (1989) presented new non-LTE models in energy balance, where the ambipolar diffusion was considered for the first time and the prominence was treated as being composed of many fine-structure threads. The thread-like models were also discussed by Heinzel (1989) and Zharkova (1989). In these papers, the effect of a mutual radiative interaction on Lyman line profiles was investigated.

The abovementioned papers form a firm basis for systematic non-LTE modelling of quiescent prominences and thus Gouttebroze et al. (1993) undertook the tedious task of constructing an extensive grid of models which could be used for comparisons with observations, particularly with expected data from the SOHO mission. This grid contains 140 isothermal-isobaric models (note that not all of them have been published in A\&AS, but they can be found in the corresponding IAS reprint). Particularly interesting are tabulated profiles and intensities of the first three Lyman lines, $\mathrm{L} \alpha, \mathrm{L} \beta$ and $\mathrm{L} \gamma$, together with the Lyman continuum. We will refer to other details later when discussing SOHO/SUMER data. All 140 models were then 
used to construct various kinds of correlations between the optical and physical properties of the models (Heinzel et al. 1994) - we will also discuss them later.

Consistent models of PCTR in energy balance were then computed by Fontenla et al. (1996), who considered a cool $(T=6000-8000 \mathrm{~K})$ fine-structure thread, surrounded by a PCTR with the temperature increasing up to $10^{5} \mathrm{~K}$. Due to effects of ambipolar diffusion, these multithread models have predicted the $\mathrm{L} \beta$ intensity much higher as compared to all previous models and even higher than observed by OSO-8. Four isobaric models with gas pressures $0.02,0.05,0.1$ and $0.2 \mathrm{dyn}^{\mathrm{cm}^{-2}}$ were considered for individual threads and then up to 100 threads were added along the line of sight. The validity of such models is to be checked against the new SOHO data.

Recently, Anzer \& Heinzel (1999 - AH) considered a new class of models which are in magnetohydrostatic equilibrium and possess a PCTR with an empirical temperature profile. It was shown that the central parts of the cool structures require an extra heating which has not so far been properly considered. In the PCTR, the radiative losses are dominated by $\mathrm{L} \alpha$ and thus the proper understanding of the formation of this line is crucial for energybalance studies. The work also predicts the intensities of $\mathrm{L} \alpha$ and $\mathrm{L} \beta$, together with $\mathrm{H} \alpha$.

Finally, higher lines of the hydrogen Lyman series were already analyzed using the SUMER/SOHO data and these first studies also include detailed non-LTE computations of their profiles, using a 12-level hydrogen model atom (Heinzel et al. 1997; Schmieder et al. 1998; SHVR). These first studies revealed a reasonable agreement between observations and theory, provided that a PCTR temperature structure is considered. However, it was noted that the higher Lyman lines are less sensitive to the PCTR structure and that more information is expected from the analysis of lower members of the series.

\section{Basic characteristics of the observed spectra and comparison with existing models}

We note some strongly asymmetric profiles and even some profiles completely shifted (March 23). These are the signatures of highly dynamical phenomena which need a careful study before assigning values of the Doppler shifts, in order to completely understand if it is a bulk flow or complex motions of plasma at different temperatures (Gontikakis et al. 1997). However, that is not the aim of this paper. The primary objective here is to see how this complete set of all Lyman lines plus continuum compares with previous observations (namely those by OSO-8) and with existing non-LTE model predictions.

\subsection{Line profiles}

In Figs. 6-8 we can recognize three rather different types of Lyman line shapes and intensities. The first two prominences (May 28 and June 2) have relatively high integrated intensities (more or less similar, namely for higher lines), but the line shapes are quite different: lines of the first prominence are in emission, while the second prominence has all lines with reversal which is very strong for the first members of the series. As we shall see later, this provides important constraints on the models. The third prominence (March 23) has lower intensities and the lower lines are reversed while higher ones are not. However, these profiles are highly asymmetrical and therefore less suitable for our discussion which is based entirely on static models. Note also that several Lyman lines are blended with lines of other species (Curdt et al. 2001). We can see this clearly in the case of L8 which has systematically somewhat higher intensity due to N IV line blend. Some other Lyman lines are blended with O I lines, but this does not affect their intensities greatly.

\section{Lo:}

The observed intensities (Fig. 6) are in general agreement with theoretical calculations. However, the details of this profile are affected by the attenuator as mentioned above and thus we cannot rely on them. A reliable reduction procedure has to be developed to take into account the influence of the attenuator. Some tests have been recently made and this work is in progress.

\section{$\mathbf{L} \boldsymbol{\beta}$ :}

Let us compare the SUMER and OSO- 8 profiles of the $\mathrm{L} \beta$ line. First, the average peak and central values of the "first and last parts of the orbit" (Fig. 4 in Vial 1982a) are respectively: 2.1 and $1.210^{-10} \mathrm{erg} / \mathrm{s} / \mathrm{cm}^{2} / \mathrm{sr} / \mathrm{Hz}$. Our values are 1.9 and $1.310^{-10}$ respectively for the March 23 prominence. The agreement is remarkably good. The slightly larger OSO-8 reversal could be due to insufficient correction of the geocoronal absorption profile; but it can also be due to the large range of observed values. The prominence of May 28 has very high central intensities (no reversals), reaching values of $7-8$ in our units (Fig. 6). The ratio of prominence to disk integrated intensities as measured by SUMER is commonly smaller than the 0.5 value found with OSO- 8 , but not for the May 28 prominence.

As mentioned above, $\mathrm{L} \beta$ poses a long-standing problem when compared with non-LTE model predictions. Typical OSO-8 integrated intensities are around 400-500 $\mathrm{erg} / \mathrm{s} / \mathrm{cm}^{2} / \mathrm{sr}$, while most of the isothermalisobaric models predict much lower values (GHV). On the contrary, Fontenla et al. (1996) obtained intensities much higher, even for models having lowest pressures. Note that the $\mathrm{L} \beta$ intensity is sensitive to the filamentary structure, i.e. to the number of threads along the line of sight, namely the line peaks (see Fontenla et al. 1996; SHVR and present models). The SUMER values of the integrated intensity are in the range given by Table 3 (see also Fig. 6), i.e. reaching $800 \mathrm{erg} / \mathrm{s} / \mathrm{cm}^{2} / \mathrm{sr}$. In particular, we can see a rather low intensity in a bubble and high intensities in low-lying prominences (Table 3). The integrated intensities in observed prominences lie between 1 and less than $1 / 3$ of the disk integrated intensity. 


\section{Higher Lyman lines:}

These are less reversed and highest members of the Lyman series show almost no reversal. Their intensities are consistent with those reported by SHVR. As we have already demonstrated in the previous paper (SHVR), the line profiles and integrated intensities of higher Lyman lines are rather sensitive to temperature and thus a PCTR must be considered to analyze these UV lines. In SHVR we have also concluded that in order to describe the behaviour of the lower part of the PCTR, one has to add the lower Lyman lines, including $\mathrm{L} \alpha$.

An example of $L \delta$ raster profiles, i.e. the profiles registered subsequently at different heights, is given in Fig. 5. At first sight this resembles theoretical height variations computed for Lyman lines from 2D models (Paletou 1995). However such variations could also be due to prominence inhomogeneities (see Fig. 4).

\section{Lyman continuum:}

The averaged intensity in the range $905-907 \AA$ is about $4.510^{-12} \mathrm{erg} / \mathrm{s} / \mathrm{cm}^{2} / \mathrm{sr} / \mathrm{Hz}$. This value is comparable to most GHV results. If we compare the average intensity at the head of the continuum with the predictions of the 140 GHV models, one can conclude that it is in general agreement with models having low temperatures (less than

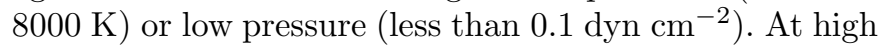
temperatures, the thickness of the layer must be small. One can note that the slope of the Lyman continuum can be an interesting diagnostic tool for the prominence temperature (see GHV and Ofman et al. 1998).

\section{$\mathbf{H} \alpha$ :}

$\mathrm{H} \alpha$ line profiles observed in the May 28 prominence have almost symmetrical Gaussian shapes and their integrated intensities are given in Table 3. The accuracy of the calibration is estimated to be $20-30 \%$ and this is due to a rather low signal/noise ratio - the prominence was relatively faint in this line. Also the calibration curve was based only on two intensity points (filters). With a better spatial resolution one could expect a higher intensity (because of a small filling factor), but this would automatically lead to line profile saturation, which is not observed. As we will see later, our measured integrated intensity of the $\mathrm{H} \alpha$ line is in very good agreement with the computed one.

\subsection{Correlations between the integrated line intensities}

Using the integrated line intensities listed in Table 3, we were first interested to see whether they can be reproduced by standard isothermal-isobaric models. We applied the procedure of Heinzel et al. (1994) to correlate theoretical integrated intensities of various lines. The observed intensities of $\mathrm{L} \alpha, \mathrm{L} \beta$ and $\mathrm{L} \gamma$ fit into the correlation plots constructed from all GHV models, but the observed points lie within points having a kinetic temperature between 10000 and $15000 \mathrm{~K}$ or perhaps even more (the maximum temperature of GHV models is $15000 \mathrm{~K}$ ). This is shown in Figs. 9a,b. Such a behaviour already indicated to us that models with a temperature variation are needed. For the prominence of May 28 we have simultaneous $\mathrm{H} \alpha$ spectra and the correlations between $\mathrm{L} \alpha$ and $\mathrm{H} \alpha$ or between $\mathrm{L} \beta$ and $\mathrm{H} \alpha$ also indicate a reasonable fit if the prominence has regions with temperatures exceeding $15000 \mathrm{~K}$ (Figs. 9c,d).

However, the problem with isothermal-isobaric models is that although the integrated intensities are in a reasonable agreement with the observed ones, a closer inspection of corresponding line profile shapes shows certain discrepancies. This is for example the case of the May 28 prominence. The models which actually correspond to points in Figs. 9c,d closest to observations (stars) have extremely reversed Lyman line profiles - see e.g. the model for $T=15000 \mathrm{~K}, p=0.1 \mathrm{dyn}^{-2}, D=5000 \mathrm{~km}$ ( $p$ is the gas pressure, $D$ the prominence thickness) in the GHV tables. Apart from L $\alpha$, the observed profiles have, on the other hand, no reversal - see Fig. 6. This thus means that we need completely different models for this particular prominence. In the following we suggest use of models with PCTR temperature gradients and with magnetohydrostatic equilibrium (non-isobaric models).

\section{Models for the PCTR}

Apart from a partial success in reproducing some individual profiles or other spectral features by existing models, it is now evident that isothermal-isobaric models, as well as multithread models with ambipolar diffusion, are hardly capable of reproducing the whole Lyman series (plus continuum and Balmer lines). Taking the prominence of May 28 as an example, it is intuitively clear that to produce high-intensity profiles which are unreversed will require not only a substantial PCTR temperature increase, but also a simultaneous decrease of the gas pressure. We remind the reader that GHV isobaric models at higher temperature can in principle predict such high intensities, but the profiles are unrealistically reversed. A class of models which considers both temperature as well as pressure variations within the PCTR was recently suggested by AH and we will now see how such models compare to SUMER observations.

\subsection{Pressure equilibrium and PCTR temperature structure}

We consider here vertical 1D-slab models as described in AH. These models have a PCTR and in general they are not isobaric. Instead, the pressure balance corresponds to the KS-type models (see Kippenhahn \& Schlüter 1957). The gas pressure $p$ varies with the column mass as

$p(m)=4 p_{\mathrm{c}} \frac{m}{M}\left(1-\frac{m}{M}\right)+p_{0}$.

The column mass $m$ extends from $m=0$ at one surface of the $1 \mathrm{D}$ slab to $m=M$ at the other, $p_{0}$ is the gas pressure at the outer boundaries. For $M$ we have

$M=\frac{B_{x} B_{z 1}}{2 \pi g}$, 

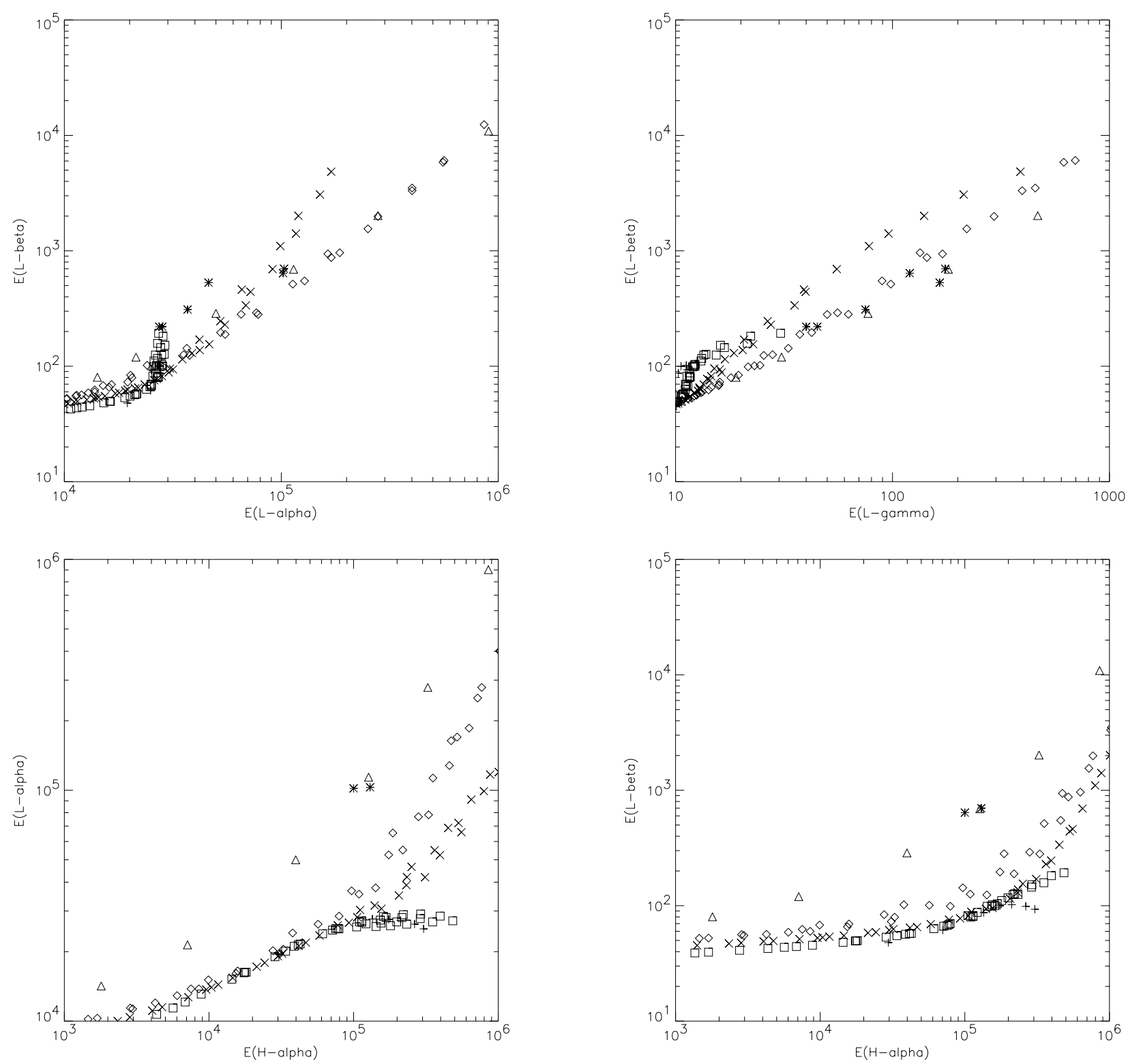

Fig. 9. Correlations between the integrated intensities of the first three Lyman lines and $\mathrm{H} \alpha$, based on isothermal-isobaric GHV models. Observed values were taken from Table 3. Observations: *, theoretical points: + $4700 \mathrm{~K}, \square 6000 \mathrm{~K}$, $\times 8000 \mathrm{~K}, \diamond 10000 \mathrm{~K}, \triangle 15000 \mathrm{~K}$

where $B_{x}$ and $B_{z 1}$ are the components of the magneticfield vector at the slab boundary, $g$ is the gravitational acceleration. $B_{x}$ is taken to be constant in KS models, $B_{z}$ varies form zero at the slab center to $B_{z 1}$ at the surface, $B_{y}=0 . p_{\mathrm{c}}$ is defined as

$p_{\mathrm{c}}=\frac{B_{z 1}^{2}}{8 \pi}$.

The central gas pressure is thus

$$
p_{\text {cen }}=p_{\mathrm{c}}+p_{0}
$$

and the mean gas pressure corresponds to

$\bar{p}=\frac{2}{3} p_{\mathrm{c}}+p_{0}$.
These equations, together with the temperature structure, allow us to evaluate the gas density $\rho$, and finally the geometrical thickness $D$ of the slab. For further details the reader is referred to $\mathrm{AH}$.

The construction of prominence models in energy balance was recently discussed by $\mathrm{AH}$, who concluded that an extra heating is needed in cool central parts in order to explain the temperature which typically exceeds the one corresponding to the radiative balance. However, the heating mechanism is not well understood although some possibilities have been suggested (Anzer \& Heinzel 2000). Here we construct a semiempirical temperature structure of the PCTR, by fitting the observed hydrogen Lyman 
spectrum. As in AH, an empirical temperature curve is approximated by the relation

$T=T_{\text {cen }}+\left(T_{\text {tr }}-T_{\text {cen }}\right)\left[1-4 \frac{m}{M}\left(1-\frac{m}{M}\right)\right]^{\gamma}$,

where $T_{\text {cen }}$ is the central temperature, $T_{\mathrm{tr}}$ the temperature at the outer boundary. The parameter $\gamma$ is used to adjust the temperature gradient in the PCTR. The higher $\gamma$, the steeper the gradient will be.

From the above equations we have the following set of adjustable (free) parameters: $T_{\text {cen }}, T_{\mathrm{tr}}, \gamma, \bar{p}, p_{0}, M$. Note that $\bar{p}$ can be replaced by $p_{\mathrm{c}}$ or $p_{\text {cen }}$. Instead of specifying these pressures, one can use the parameter $B_{z 1}$ or $B_{x}$; they are related through $M$ (Eq. (2)). Other free parameters which are important for line profile computations are the microturbulent velocity $v_{\mathrm{t}}$ and the number of slabs along the line of sight.

We stress that the pressure equilibria described above correspond to the situation when one looks along the magnetic-field lines. The opposite case, equally important for prominence modelling, takes place when we look in a direction across the field lines. In such a situation, however, individual plasma threads are magnetically isolated and thus their pressures and temperatures can have rather arbitrary variations. As a first approximation, one representative thread can be modelled as isobaric and almost isothermal with a step-like PCTR. This is formally achieved by setting $\bar{p}=p_{0}$. The temperature is either constant $\left(T_{\mathrm{tr}}=T_{\text {cen }}\right)$ or somewhat increasing towards the boundaries. A more general picture of $2 \mathrm{D}$ threads in magnetic equilibria is presented in Heinzel \& Anzer (2001).

Non-LTE transfer models are constructed for a 12-level plus continuum atom, using partial redistribution in $\mathrm{L} \alpha$ and $\mathrm{L} \beta$ (higher Lyman lines are less sensitive to PRD). Incident radiation fields (i.e. the boundary conditions) and the computational methods are described in Heinzel et al. (1997), SHVR and AH (see also the references therein). We consider 1D models, but distinguish between the line of sight along the field lines and across them. The synthetic intensities are finally computed for 1, 10 and 30 finestructure threads, using the standard approach of identical threads (Heinzel 1989; Vial et al. 1989).

\subsection{Results of numerical modelling}

The following non-LTE computations are aimed at explaining the newly observed features of the Lyman series, but they do not pretend to fit the line intensities in all details. First, a strong constraint comes from the L $\alpha$ line which is unfortunately not as accurate as other lines of the series (due to instrumental effects of the attenuator). Second, the spectra are averaged over space and time and are not taken simultaneously. Finally, we have found it very difficult to fit all Lyman lines plus continuum (and $\mathrm{H} \alpha$ ) just by trial-and-error methods and more sophisticated optimization or grid techniques will be needed for this task.
Table 4. Lyman decrement (defined as the ratio of the line integrated intensity to that of $\mathrm{L} \beta$ ). Bottom part gives $\mathrm{L} \beta$ and $\mathrm{H} \alpha$ integrated intensities $\left(\mathrm{erg} / \mathrm{s} / \mathrm{cm}^{2} / \mathrm{sr}\right)$ and the Lyman continuum specific intensity $\left(\mathrm{erg} / \mathrm{s} / \mathrm{cm}^{2} / \mathrm{sr} / \mathrm{Hz}\right)$. Observed Lymanline intensities correspond to the mean values from Figs. 6-8. All theoretical values correspond to PRD models; 10 threads were added along the line of sight for June 2 prominence

\begin{tabular}{llllll}
\hline Line & $\begin{array}{l}\text { May } 28 \\
\text { obs. }\end{array}$ & theor. & $\begin{array}{l}\text { June } 2 \\
\text { obs. }\end{array}$ & theor. & $\begin{array}{l}\text { March 23 } \\
\text { obs. }\end{array}$ \\
\hline L $\alpha$ & 127 & 90.3 & 84 & 121 & 119 \\
$\mathrm{~L} \beta$ & 1 & 1 & 1 & 1 & 1 \\
$\mathrm{~L} \gamma$ & 0.22 & 0.29 & 0.30 & 0.15 & 0.25 \\
$\mathrm{~L} \delta$ & 0.10 & 0.17 & 0.16 & 0.085 & 0.13 \\
$\mathrm{~L} \epsilon$ & 0.050 & 0.13 & 0.071 & 0.079 & 0.068 \\
$\mathrm{~L} 6$ & 0.032 & 0.094 & 0.046 & 0.063 & 0.045 \\
$\mathrm{~L} 7$ & 0.022 & 0.072 & 0.036 & 0.056 & 0.034 \\
$\mathrm{~L} 8$ & 0.030 & 0.058 & 0.042 & 0.050 & 0.042 \\
$\mathrm{~L} 9$ & 0.020 & 0.048 & 0.030 & 0.046 & 0.029 \\
\hline $\mathrm{L} \beta$ & 791 & 750 & 553 & 609 & 312 \\
$\mathrm{H} \alpha$ & $1.3+5$ & $1.72+5$ & & $7.3+5$ & \\
$\mathrm{~L}-\mathrm{cont}$ & $0.61-11$ & $0.95-11$ & $0.45-11$ & $0.96-11$ & $0.45-11$ \\
\hline
\end{tabular}

\subsubsection{Prominence of May 28}

As we saw in Sect. 2, different prominences show a large variety of profiles and intensities (including the Lyman continuum intensity) and this is also the case inside one particular prominence (March 20) as shown in Fig. 5. The most complete set of observations is that of May 28, which also includes the $\mathrm{H} \alpha$ line. We therefore focus on these data and try to find an optimal PCTR model. Our interest in this particular prominence is further strengthened by the fact that we detect very high intensities and simultaneously all lines (except for L $\alpha$ ) are unreversed which cannot be explained by simple isothermal-isobaric models as shown above. After trial-and-error modelling we found a reasonable model (Fig. 10) with its intensities displayed in Fig. 11 and the Lyman decrement given in Table 4. For comparison, we show in Table 4 also the observed Lyman decrements. While the radiometric calibration has relative uncertainties of $30-40 \%$, the line ratios are accurate to within $5-10 \%$, depending on the count statistics (W. Curdt and K. Wilhelm - private communication).

The model was constructed in such a way that the $\mathrm{L} \beta$ integrated intensity fits the mean observed value taken from Fig. 6 to within 10\%. Also the line profile itself reproduces the observed one rather well, providing that the microturbulent velocity in the PCTR is around $20 \mathrm{~km} \mathrm{~s}^{-1}$. In these models we keep the microturbulent velocity constant in the whole structure, which is certainly not realistic in the central parts where e.g. the $\mathrm{H} \alpha$ line is formed. The basic observed feature - all lines starting from $\mathrm{L} \beta$ in emission - is also well reproduced, although the Lyman decrement for higher lines is a factor of two larger than that observed (Table 4). Also, the L $\alpha$ integrated intensity is somewhat larger than the mean observed value, but L $\alpha$ observations are strongly influenced by instrumental 

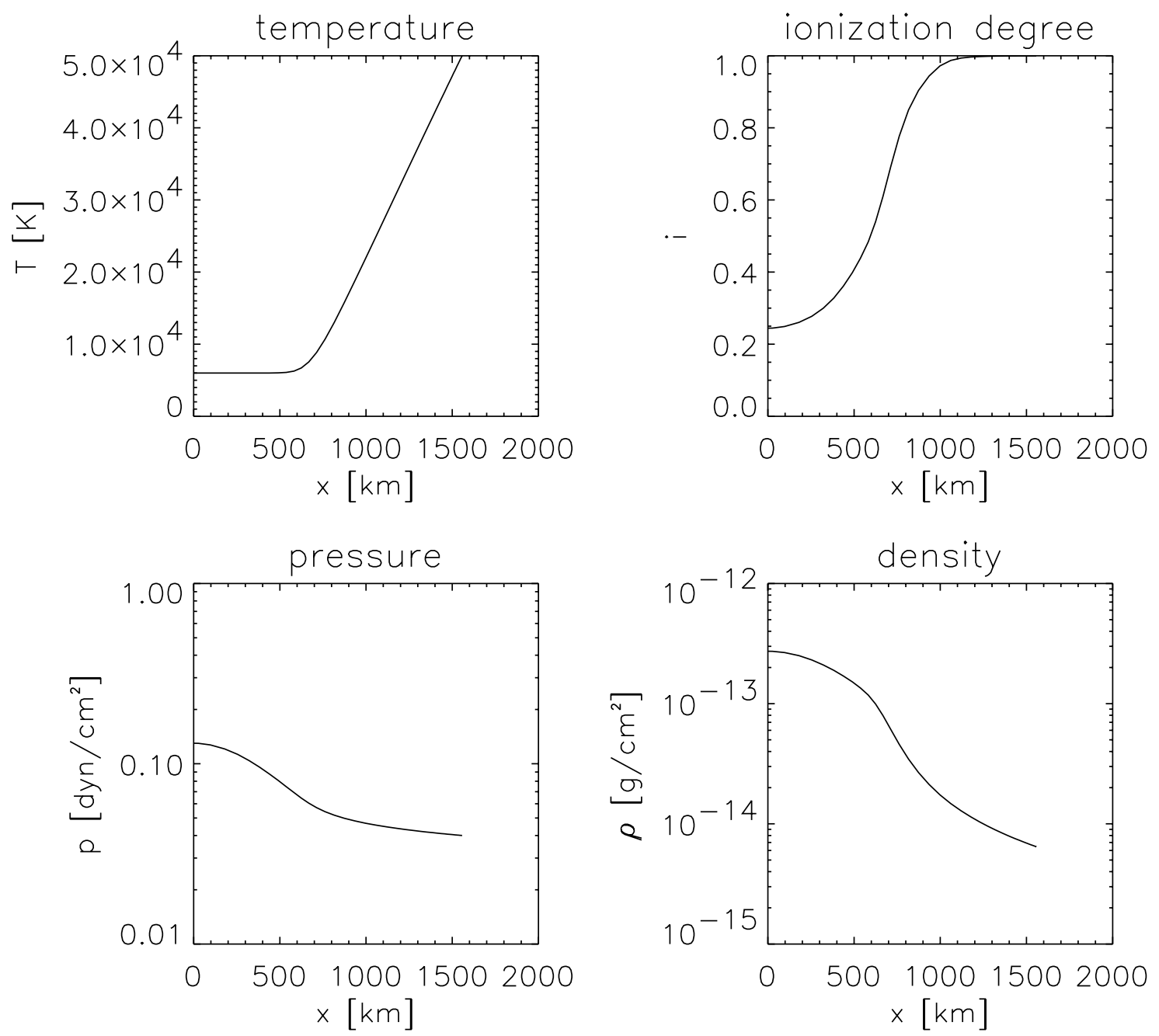

Fig. 10. May 28 prominence model with $\gamma=13$ (height $10000 \mathrm{~km}$ ). Only one half of a symmetrical 1D slab is displayed

effects. Figure 10 shows the spatial variations of different plasma parameters for the model of the May 28 prominence. Our model assumes that we see the structure more or less along the magnetic field lines. A strong temperature increase, together with pressure variations which obey the equilibrium solution, are responsible for strong emission lines without any reversal. The theoretical $\mathrm{L} \alpha$ profile has a reversal, but we do not see it clearly in the observed profile because of the distortion caused by the attenuator. However, the observed line center intensity lies well above the value of the diluted incident chromospheric intensity and this is consistent with enhanced thermal emission in the PCTR.

Finally, an interesting behaviour was found for PRD line transfer in $\mathrm{L} \alpha$ and $\mathrm{L} \beta$. The PRD profiles of these two lines are almost identical with the CRD profiles, while higher lines show some differences. $\mathrm{H} \alpha$ is however almost a factor of two brighter in the case of PRD compared to CRD. We explain this effect as follows: the emission cores of the lower Lyman lines are formed in the PCTR and thus they are dominated by a Doppler diffusion (the Lorentz wings of higher lines are not seen, compare to the prominence of June 2). Moreover, thermal emission is also enhanced within the PCTR compared to cool central parts. However, the $\mathrm{L} \alpha$ wing photon diffusion still has a partially coherent nature (wings are formed deeper) and as was demonstrated already by Heinzel et al. (1987), such a quasi-coherent penetration of boundary-layer photons leads to both electron density enhancements and an increase in the $\mathrm{H} \alpha$ integrated intensity. In the present case, the electron density in the slab center is increased for the PRD case by a factor of 1.5 relative to CRD. Since the $\mathrm{H} \alpha$ integrated intensity is proportional to $n_{\mathrm{e}}^{2}$, we get PRD brightness in this line about a factor of two larger, which is consistent with our models. Enhancement of $n_{\mathrm{e}}$ due to $\mathrm{PRD}$ in $\mathrm{L} \alpha$ is also responsible for a somewhat larger emission of higher Lyman lines, although they are computed with CRD.

\subsubsection{Prominence of June 2}

This prominence has similar or somewhat lower integrated line intensities as the previous one, but exhibits strong reversals in all Lyman lines. Contrary to the previous model, 

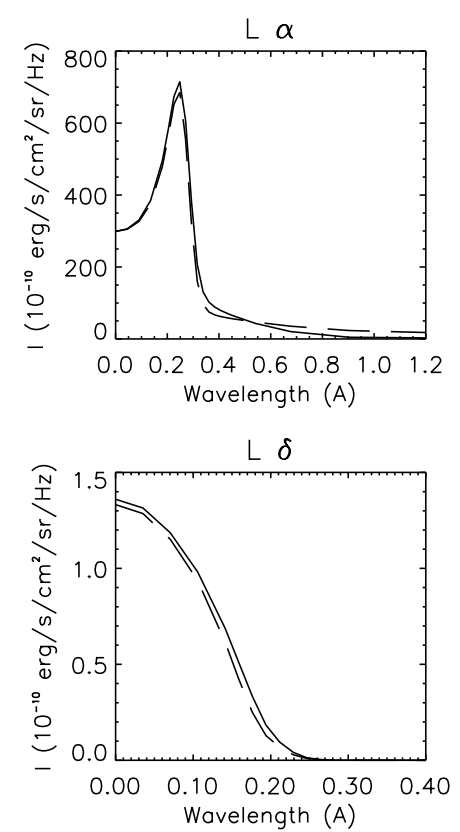

L 7

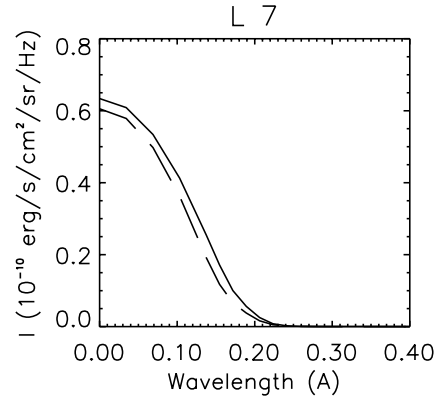

$\mathrm{H} \alpha$

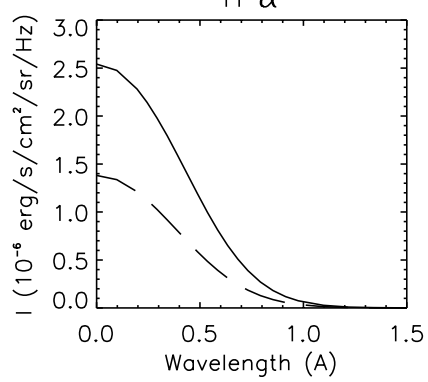

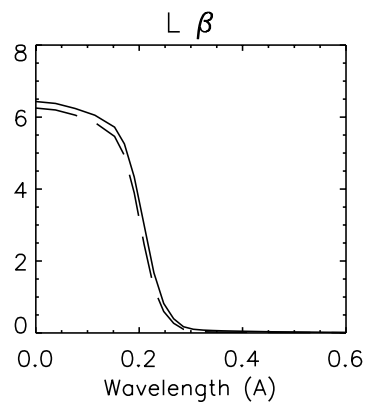

$L \epsilon$

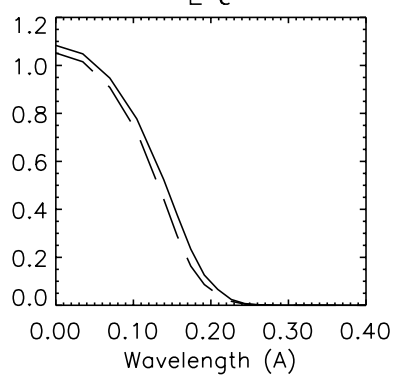

L 8

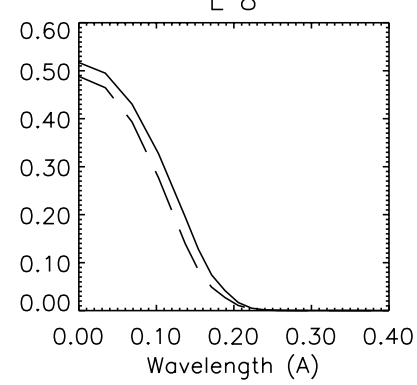

$H \beta$

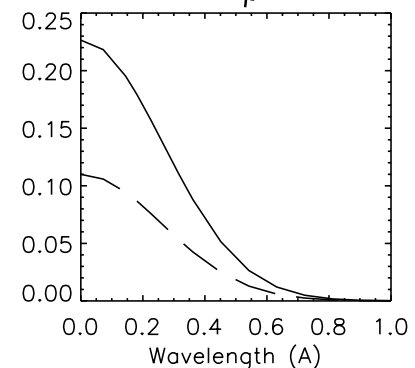

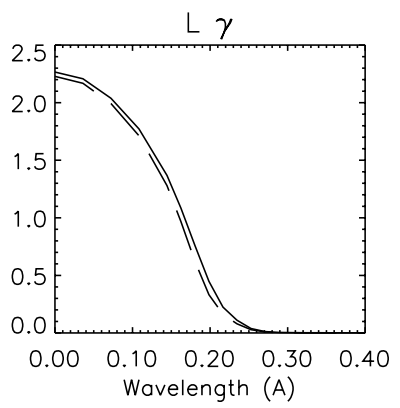

L 6

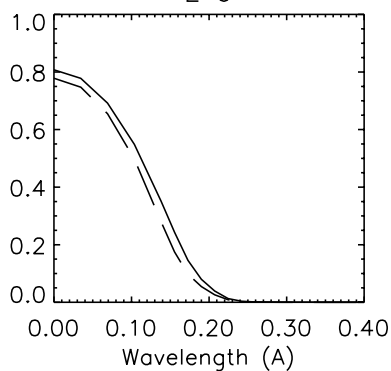

L 9

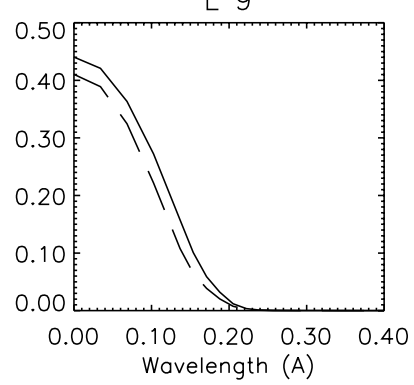

$\mathrm{H} \gamma$

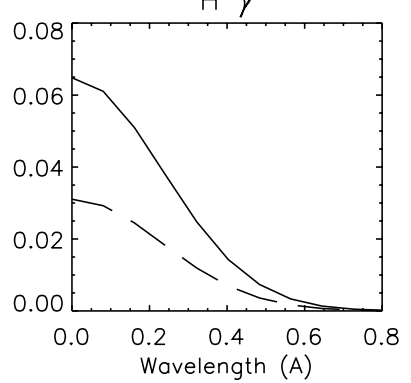

Fig. 11. May 28 - PRD(full)/CRD(dash) synthetic profiles

the reversed profiles can be obtained for a class of models which do not have a significant temperature increase (even isothermal GHV models give a rough solution) and which are practically isobaric. Such a solution is shown in Fig. 12, the Lyman decrement is again given in Table 4. The synthetic line profiles are displayed in Fig. 13.

Closer inspection of the observed Lyman profiles reveals a certain asymmetry but this varies along the whole Lyman series and even changes from red to blue. We thus conclude that this asymmetry lies within the data accuracy and does not result from systematic flows (contrary to the case of the March 23 prominence). However, the profiles are rather broad and this cannot be accounted for by an increased microturbulent velocity (which would then exceed the sound speed). But as we see in Fig. 13, these reversed profiles are very sensitive to the number of fine-structure threads along the line of sight and by taking 10-30 threads, we are able to fit the line widths. PRD effects are critical for $\mathrm{L} \alpha$ and $\mathrm{L} \beta$ and the line profiles markedly differ from those computed with CRD. For isothermal-isobaric slabs this behaviour was demonstrated by Heinzel et al. (1987). Both PRD and CRD, as well as the observed $\mathrm{L} \alpha$ line-center intensities are at a level of the diluted incident chromospheric radiation which supports our conclusions that the PCTR doesn't play an important role and the Lyman lines are thus rather sensitive to the incident radiation fields. In fact, our interpretation is that we look mainly across the magnetic-field lines and in 

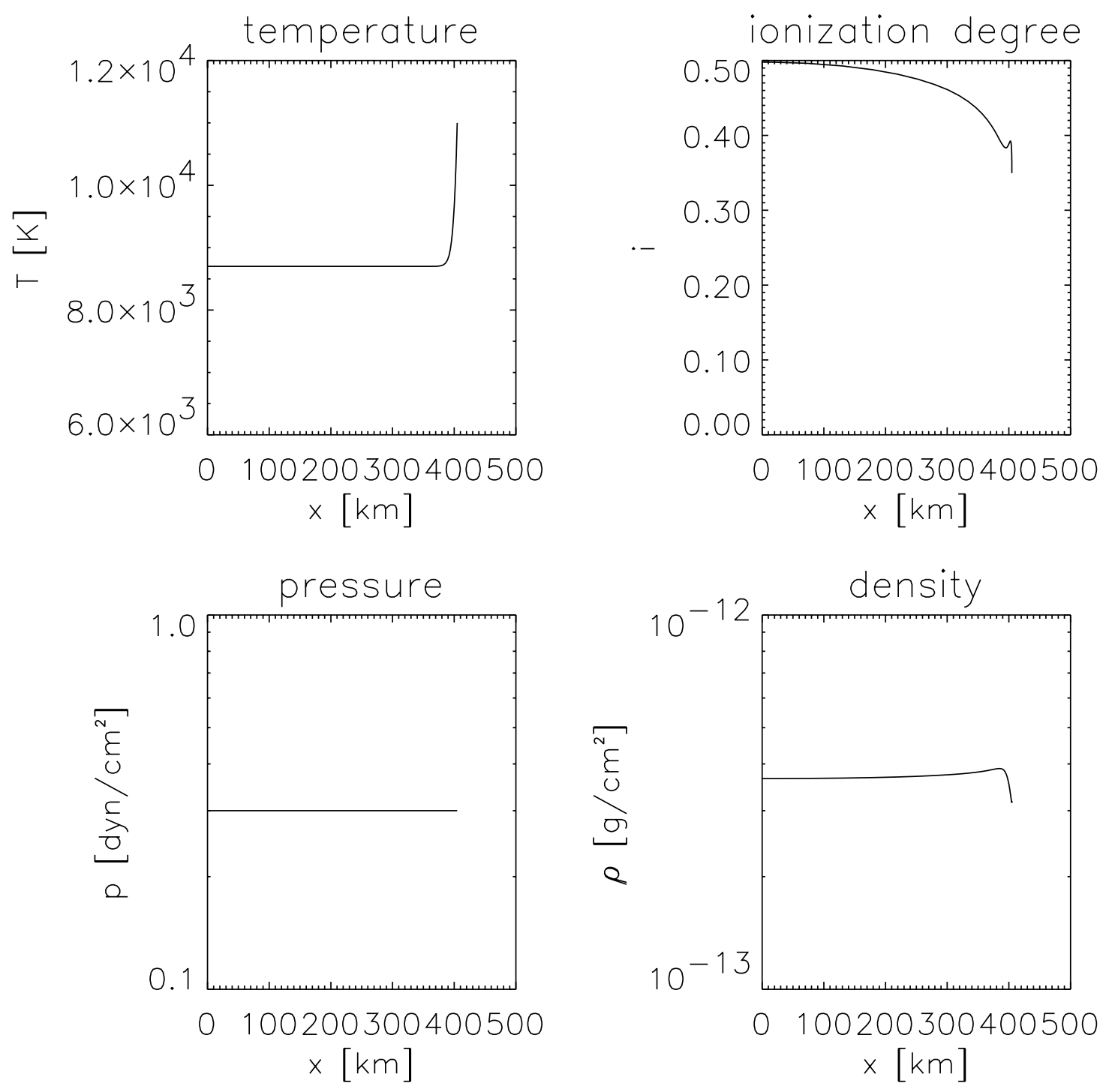

Fig. 12. June 2 prominence model with $\gamma=40$ (height $25000 \mathrm{~km}$ ). Only one half of a symmetrical $1 \mathrm{D}$ slab is displayed

such a case the PCTR will be extremely narrow with very steep temperature gradients. We see several mostly cool condensations which are thermally isolated from the hot corona by the magnetic field (heat conduction across the magnetic field is neglected). However, the same structures when viewed along the field lines must have PCTR of the type discussed in the previous subsection.

\section{Conclusions}

The set of Lyman lines was observed in several prominences by the SOHO/SUMER spectrograph. For the first time we present quasi-simultaneous observations of both lower and higher members of the hydrogen Lyman series, together with the Lyman continuum. For the prominence of May 28, the $\mathrm{H} \alpha$ spectrum was also obtained at Ondřejov observatory. The observed prominences show a large variety of Lyman line profiles and integrated intensities. In one case, all profiles (except $\mathrm{L} \alpha$ ) are unreversed; other prominences show reversed profiles, at least for the lower members of the series. However, it is interesting to note that the Lyman decrement is rather similar for the three observed prominences.

We have attempted to interpret the Lyman lines observed in two quiescent prominences (May 28 and June 2). For this we used models suggested by Anzer \& Heinzel (1999). In the case of the May 28 prominence, a PCTR which forms along the magnetic-field lines is necessary to explain strong and mostly unreversed lines. We were able to fit the $\mathrm{L} \beta$ line which exhibits strong emission (no reversal), but the computed intensities of higher lines are somewhat larger than the observed ones. We conclude that we see one slab (thread) almost along the field lines. On the other hand, the June 2 prominence has strongly reversed profiles and this can be explained by an almost isobaric model without any significant temperatue rise. Such a situation is possible when we look more or less perpendicularly to the field lines and see several threads along the line 

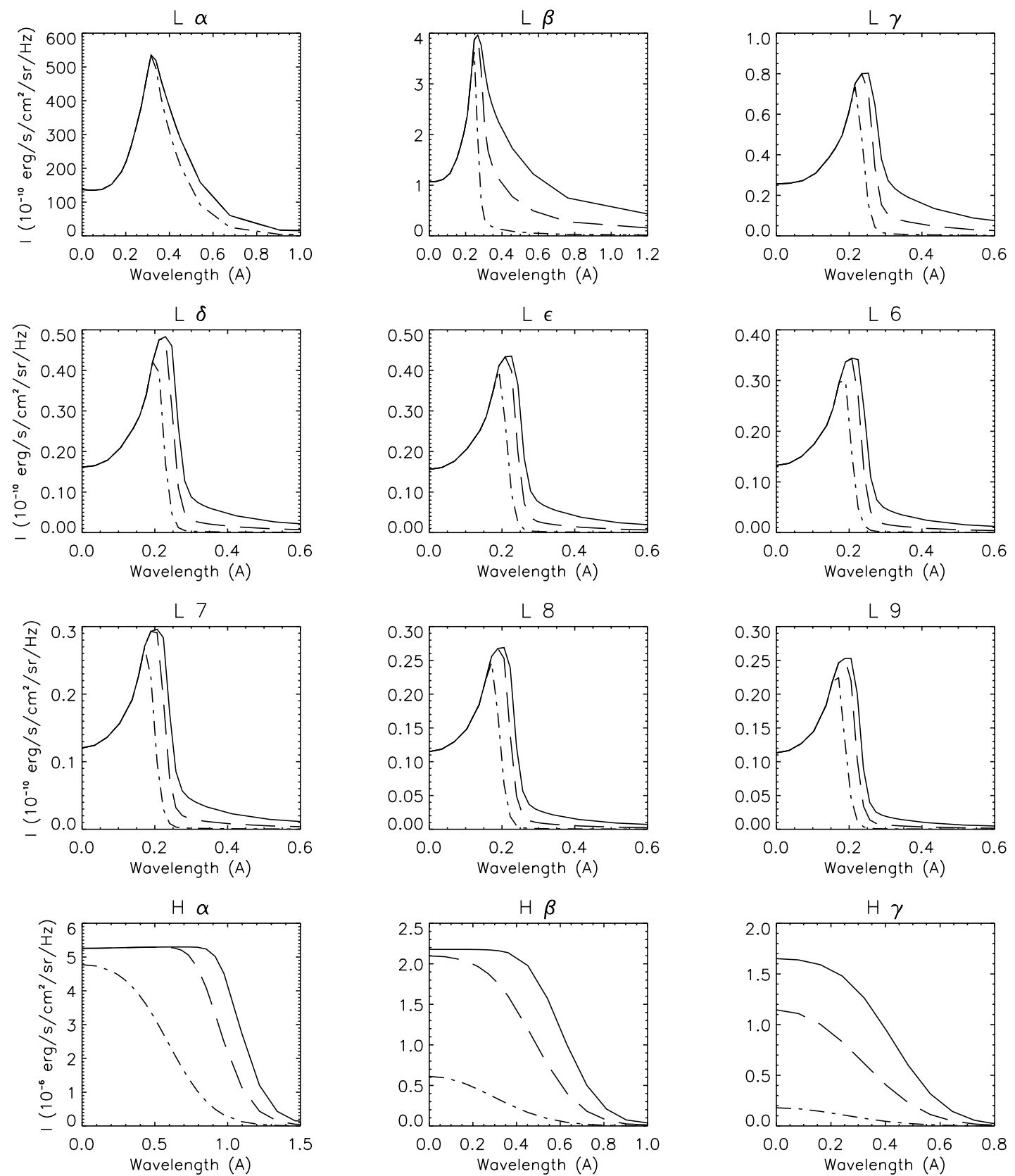

Fig. 13. June 2 - PRD synthetic profiles. Full: 30 threads, dash: 10 threads, dot-dash: 1 thread

of sight. These threads are magnetically isolated and thus do not show any contributions from presumably narrow PCTR. However, the higher lines are again more intense than is actually observed. Our trial-and-error line fitting is certainly inadequate to obtain an optimum solution for all lines. Nevertheless, apart from certain discrepancies which are also partly due to averaging, calibration and blends, the quantitative agreement between SUMER spectra and synthetic ones based on sophisticated models is encouraging.

Concerning the GHV grid of isothermal-isobaric models, these models predict rather well the intensities of optical lines formed in the central parts of prominences at low temperatures (for example Balmer lines). They may also give us some insight into the behaviour of structures seen across the field lines. However, all such models fail completely when the PCTR (along the field lines) plays the role as in the case of the May 28 prominence.

Our general conclusion is that both observed prominences, although having quite different line profiles, can be modelled in the frame of $2 \mathrm{D}$ magnetohydrostatic equilibria. The corresponding temperature structures are very different; PCTR increase in one case and almost an isothermal situation in the other. Therefore, two quite different boundary layers are probably seen when viewing the prominence along and across the field lines, respectively, and our SOHO/SUMER observations seem to indicate such a behaviour. These models can explain the 
vertical threads in almost horizontal but dipped magnetic fields. In that respect, further observations of Lyman series (together with other UV lines) are needed which will help us to better understand the $2 \mathrm{D}$ or $3 \mathrm{D}$ nature of the prominence fine structure. Theoretical modelling has to be extended to fully 2D or 3D magnetohydrostatic equilibria in which the radiation is treated in the frame of $2 \mathrm{D}(3 \mathrm{D})$ non-LTE transfer (see Heinzel \& Anzer 2001).

Acknowledgements. The SUMER project is financially supported by DLR, CNES, NASA, and the ESA PRODEX programme (Swiss contribution). SUMER is on-board the SOHO spacecraft, a joint project of ESA and NASA. P.H. and P.K. acknowledge the support from CNRS and from the grants No. A3003902 and S-1003006 of the Academy of Sciences of the Czech Republic and No. 205/00/1726 of GACR. We want to thank particularly the SUMER planners T. Kucera, P. Lemaire and K. Wilhelm for their help during observations. Numerous discussions with U. Anzer are highly appreciated.

\section{References}

Anzer, U., \& Heinzel, P. 1999, A\&A, 349, 974 (AH)

Anzer, U., \& Heinzel, P. 2000, A\&A, 358, L75

Curdt, W., Brekke, P., Feldman, U., et al. 2001, A\&A, in press

Fontenla, J. M., Reichman, E. J., \& Tandberg-Hanssen, E. 1988, ApJ, 329, 464

Fontenla, J. M., Rovira, M., Vial, J.-C., \& Gouttebroze, P. 1996, ApJ, 466, 496

Gontikakis, C., Vial, J. C., \& Gouttebroze, P. 1997, A\&A, 325, 803

Gouttebroze, P., Heinzel, P., \& Vial, J.-C. 1993, A\&AS, 99, 513 (GHV)

Harrison, P., Sawyer, E. C., et al. 1995, Solar Phys., 162, 233

Heasley, J. N., \& Milkey, R. W. 1978, ApJ, 221, 677

Heasley, J. N., \& Milkey, R. W. 1983, ApJ, 268, 398

Heinzel, P. 1989, Hvar Obs. Bull., 13, 279

Heinzel, P., \& Anzer, U. 2001, ApJ, submitted

Heinzel, P., Gouttebroze, P., \& Vial, J.-C. 1987, A\&A, 183, 351
Heinzel, P., Gouttebroze, P., \& Vial, J.-C. 1988, in Dynamics and Structure of Solar Prominences, Proc. of a Workshop held at Palma (Mallorka), ed. J. L. Ballester, \& E. R. Priest (Universitat de les Illes Balears), 71

Heinzel, P., Gouttebroze, P., \& Vial, J.-C. 1994, A\&A, 292, 656

Heinzel, P., Schmieder, B., \& Vial, J.-C. 1997, in Proc. SOHO 5 Workshop, ESA SP-404, 427

Kippenhahn, R., \& Schlüter, A. 1957, Z. Astrophys., 43, 36 (KS)

Kotrč, P. 1997, Hvar Obs. Bull., 21, 97

Kucera, T., Andretta, V., \& Poland, A. 1998, Solar Phys., 183, 107

Ofman, L., Kucera, T., Mouradian, Z., \& Poland, A.I. 1998, Solar Phys., 183, 97

Paletou, F. 1995, A\&A, 303, 587

Paletou, F., Vial, J.-C., \& Auer, L. H. 1993, A\&A, 274, 571

Poland, A. I., \& Tandberg-Hanssen, E. 1983, Solar Phys., 84, 63

Schmieder, B., Heinzel, P., Kucera, T., \& Vial, J.-C. 1998, Solar Phys., 181, 309

Schmieder, B., Heinzel, P., Vial, J.-C., \& Rudawy, P. 1999a, Solar Phys., 189, 109 (SHVR)

Schmieder, B., Kucera, T., Heinzel, P., \& Vial, J.-C. 1999b, in Proc. SOHO 8 Workshop, ESA SP-446, 605

Schmieder, B., Kotrč, P., Heinzel, P., Kucera, T., \& Andretta, V. 1999c, in Proc. 9th European Solar Physics Meeting, ESA SP-448, 439

Schühle, U., Hollandt, J., Pauluhn, A., \& Wilhelm, K. 2000, ESA SP-463, 427

Vial, J.-C. 1982a, ApJ, 253, 330

Vial, J.-C. 1982b, ApJ, 254, 780

Vial, J.-C., Rovira, M., Fontenla, J., \& Gouttebroze, P. 1989, Hvar Obs. Bull., 13, 347

Wiik, J. E., Dammasch, I. E., Schmieder, B., \& Wilhelm, K. 1999, Solar Phys., 187, 405

Wilhelm, K., Curdt, W., Marsch, E., et al. 1995, Solar Phys., 162,189

Wilhelm, K., Lemaire, P., Curdt, W., et al. 1997, Solar Phys., 17075

Zharkova, V. V. 1989, Hvar Obs. Bull., 13, 331 